\section{PSICOLOGİA y EDUCACIÓN}

\author{
Revista Intercontinental de Psicología y \\ Educación \\ ISSN: 0187-7690 \\ ripsiedu@uic.edu.mx \\ Universidad Intercontinental \\ México
}

Palacios Delgado, Jorge Raúl; Sánchez Torres, Berenise; Andrade Palos, Patricia Intento de suicidio y búsqueda de sensaciones en adolescentes

Revista Intercontinental de Psicología y Educación, vol. 12, núm. 1, enero-junio, 2010, pp. 53-75 Universidad Intercontinental

Distrito Federal, México

Disponible en: http://www.redalyc.org/articulo.oa?id=80212393004

Cómo citar el artículo

- Número completo

- Más información del artículo

- Página de la revista en redalyc.org

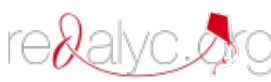

Sistema de Información Científica

Red de Revistas Científicas de América Latina, el Caribe, España y Portugal Proyecto académico sin fines de lucro, desarrollado bajo la iniciativa de acceso abierto 


\title{
Intento de suicidio y búsqueda de sensaciones en adolescentes
}

\author{
Jorge Raúl Palacios Delgado \\ Berenise Sánchez Torres \\ y Patricia Andrade Palos
}

\begin{abstract}
Resumen
Abstract

En los últimos años, el intento de suicidio ha aumentado en los adolescentes mexicanos, convirtiéndose en un problema de salud pública. La investigación ha demostrado que las variables de personalidad se asocian con este comportamiento. Los objetivos de la presente investigación fueron describir el intento de suicidio, determinar las diferencias en la búsqueda de sensaciones entre los adolescentes que han y no han intentado suicidarse y conocer la asociación entre la búsqueda de sensaciones y la letalidad.

In last years the suicidal behavior is a prevalent behavior in Mexican adolescents and contributes to elevated rates for many problems of health. The literature has showed the multiples causes for suicidal attempt and personality variables have demonstrated an association with this behavior. The objective of this research was analyze the differences and correlates of sensation seeking in suicidal behavior in a sample of Mexican youths. The studied sample was formed by 550 teenagers between 14 and 23 years
\end{abstract}

* Esta investigación estuvo financiada por el Programa de Apoyo a Proyectos de Investigación e Innovación Tecnológica (PAPITT) proyecto \# IN304607-2 con el nombre Recursos Psicológicos en Adolescentes.

Jorge Raúl Palagios Delgado, Berenise Sánchez Torres y Patricia Andrade Palos, Facultad de Psicología, Universidad Nacional Autónoma de México. [kobuj@yahoo.com.mx]

Revista Intercontinental de Psicología y Educación, vol. 12, núm. 1, enero-junio 2010, pp. 53-75. Fecha de recepción: 16 de julio de 2008 | fecha de aceptación: 30 de junio de 2009. 
Se seleccionó una muestra de 550 jóvenes, 260 hombres y 290 mujeres, entre 14 y 23 años, estudiantes de bachillerato de la Ciudad de México. Para medir la búsqueda de sensaciones, se utilizó una nueva escala que mide este constructo. El intento de suicidio se midió mediante un cuestionario sobre conducta suicida. Se igualó la muestra por sexo y edad, con la finalidad de que los grupos fueran equivalentes. Los resultados mostraron diferencias entre hombres y mujeres en el intento de suicidio. Los jóvenes que han intentado quitarse la vida presentan mayor búsqueda de sensaciones que los que no lo han intentado. Asimismo, se mostró una relación significativa entre el deseo de morir y la búsqueda de sensaciones. old, 260 males and 290 females, students of public high schools in Mexico City. New scale measuring sensation seeking was used. The youths completed a survey which assessed suicidal behavior. Adolescents were classified into two groups: a suicidal attempt and a non suicidal attempt. The results indicated differences between male and female in suicidal attempt. Compared with control group, the suicidal attempt group had the highest levels of sensation seeking. Was showed correlations between sensation seeking and level of lethality.

KEY WORDS

lethality

Palabras clave

letalidad

\section{Introducción}

E 1 comportamiento suicida está constituido por diferentes etapas, las cuales no son necesariamente secuenciales ni indispensables; entre ellas, puede encontrarse la ideación suicida, la contemplación activa del propio suicido, la planeación y preparación, la ejecución del intento suicida y el suicido consumado (Rivera, 2007). Pueden presentarse casos donde se haya pasado por todas las etapas para llegar a la muerte, o bien, casos donde el suicidio se consume sin preámbulos, pero la interacción de estas etapas incrementa el riesgo suicida (GonzálezForteza, Mariño, Rojas, Mondragón y Medina-Mora, 1998). 
En el presente artículo se discutirá sobre el intento de suicidio, el cual se define como un acto o conducta que realiza una persona con el objeto de atentar contra su propia existencia sin lograrlo (Rivera y Andrade, 2006). No siempre el intento o propósito suicida tiene como finalidad el hecho de quitarse la vida; una persona puede inflingirse una lesión no necesariamente para morir, sino con la finalidad de obtener ganancias secundarias, como llamar la atención o manipular. De esta forma, el individuo se pone en riesgo, pues puede morir sin habérselo propuesto o bien puede suceder lo contrario: no muere aun deseando hacerlo (González-Forteza et al.).

En México, durante los últimos años se ha incrementado el índice de intentos de suicidio y de suicidios consumados, lo cual lo convierte en un problema de salud pública. El Instituto Nacional de Estadística Geografía e Informática (INEGI, 2005) señaló que en el año 2004 se registraron 3324 intentos de suicidio, de los cuales la primera causa fue por un disgusto familiar (280 casos), la segunda fue por una dificultad amorosa (230 casos) y el tercer motivo se debió a una enfermedad grave e incurable (172 casos). En este mismo periodo, los estados que tuvieron una prevalencia mayor fueron: Veracruz (324 casos), Jalisco (316 casos), Chihuahua (192 casos) y el Distrito Federal (189 casos). Además, la mayoría de los suicidios consumados fueron realizados por jóvenes entre los 15 y 19 años (12.6\%). Al hacer el análisis por sexo, la encuesta encontró que en los hombres la mayoría tenía entre 20 y 24 años (12.3\%), seguido por los de 15 y 19 años (11.6\%). En las mujeres, la mayoría de los suicidios se ubicaron entre los 15 y los 19 años de edad (16.3\%); esta misma encuesta señala que, en comparación con las mujeres (6.3\%), los varones son quienes más lo consiguen (8.5\%).

Los reportes del intento suicida en adolescentes de la ciudad de México señalan que, en el año 1997, la proporción fue de $8.3 \%$ y para el año 2000 se incrementó a 9.5\% (González-Forteza et al., 2002). En cuanto a las diferencias por sexo, González-Forteza et al., (2002) mencionaron que en 1997 la prevalencia en las mujeres fue de $12.1 \%$ y en los hombres de $4.3 \%$, lo que representa una proporción de tres mujeres por cada hombre. En el año 2000, la prevalencia en las mujeres aumentó a $15.1 \%$ y en los 
hombres disminuyó a 3.9\%, por lo que la proporción hombre-mujer se incrementó a casi 4:1, estos mismos autores reportaron que casi una de cada cuatro mujeres con intento ya lo ha probado dos o más veces en su vida (en 1997, 23.6\% y en 2000, 25.9\%). En los hombres, se observó un incremento, pues en 1997 la proporción de intentos recurrentes fue de casi 1:5 (18.8\%) y en 2000 fue de uno por cada tres, es decir, aproximadamente $29 \%$.

La literatura indica que los hombres ejecutan el suicidio con una frecuencia de cinco veces mayor que las mujeres; asimismo, se señala que las mujeres intentan suicidarse tres o cuatro veces más frecuentemente que los hombres (Andrade, Betancourt y Camacho 2003; Cohen, Spirito y Brown, 1996; Palacios, Andrade y Betancourt, 2006). Si bien la problemática del intento suicida predomina en la población femenina, siguen siendo los varones quienes más consiguen suicidarse. Por otra parte, GonzálezForteza et al. (2002) señalaron que la edad en que se realizó el único o último intento suicida por los hombres y las mujeres fue aproximadamente a los 13 años (con rangos entre los 10 y 15 años); es decir, puede considerarse que en este tiempo cursaban los últimos años de la primaria o la secundaria, por lo que estos niveles educativos constituyen los sectores más propicios para la atención y prevención de la conducta suicida.

El intento de suicidio es multicausado. Para su estudio se han propuesto algunos modelos teóricos que explican los factores que influyen en el intento de suicidio; dentro de estos estudios, puede destacarse el modelo de riesgo suicida en adolescentes de Spirito, Overholser y Vinnick (1995); en él, los autores señalan que existen factores predisponentes, los cuales, al asociarse con algunos estresores (dificultades sociales, escolares y familiares) y con estados emocionales negativos (disforia, desesperación, enojo) influyen en conductas de afrontamiento desadaptativas y en su conjunto inciden en la conducta suicida. Otro modelo conceptual propuesto por Beautrais (1998) asume la relación entre el factor social, de personalidad y el familiar, así como la interacción entre la susceptibilidad individual (desórdenes psiquiátricos) y altos niveles de exposición a eventos de vida estresantes, quienes en su conjunto tienen una directa 
o indirecta contribución sobre el riesgo de intentar suicidarse. Stoelb y Chiriboga (1998) propusieron un modelo para medir el grado de riesgo suicida, considerando que los factores asociados a la problemática suicida pueden dividirse en: primarios (intentos previos, desórdenes afectivos y desesperanza), secundarios (el abuso de substancias, rasgos de personalidad o desórdenes conductuales) y finalmente los factores situacionales (funcionamiento familiar, exposición a suicidios, soporte social y estrés).

Dentro de los modelos explicativos en torno del intento de suicidio, un aspecto central es el efecto que tienen las variables individuales en este comportamiento, de los cuales se destacan los pensamientos, conductas y las expectativas del intento de suicidio (Brown, Henriques, Sosdjan y Beck, 2004; Nock, Holmberg, Photos y Michel, 2007); asimismo, el locus de control, la desesperanza (Spann, Molock Barksdale, Matlin y Puri, 2006) o percibirse con menos recursos (Kaslow, Okun, Young, Wyckoff, Thompson, Price, Bender, Womey, Goldin y Parker, 2002; Rivera, 2007).

La investigación ha documentado que la personalidad es un eje de análisis de los correlatos incidentes en el intento de suicidio en adolescentes. La evidencia menciona que los trastornos psicoafectivos (González-Forteza, Berenson, Tello, Facio y Medina- Mora, 1998), la ansiedad (Goldston, Reboussin y Daniel, 2006), la impulsividad (Horesh, Gothelf, Weizman y Apter, 1999), el neuroticismo (Beautrais, 1998), la extroversión (Roque, Valadez, González de Mendoza, Vega, Flores y Valencia, 2004) o rasgos de personalidad como paranoia, psicastenia o desviación psicopática (Loza, Lucio y Durán, 1998) y especialmente el rol que tiene factores intermediarios —en particular, la depresión (Goldston et al., 2006; González-Forteza, Ramos, Caballero y Wagner, 2003) — se han postulado como explicaciones del intento de suicidio.

En este sentido, Harrington (2001) considera que la conducta suicida en los adolescentes puede explicarse a partir de la combinación de la depresión con ciertas características de personalidad, tales como la agresión o la propensión a tomar riesgos. Rosenbloom (2003) menciona que se han integrado y modificado los factores de personalidad que se consideran relevantes en la presencia de conductas de riesgo, clasificando 
el rango de dimensiones de personalidad en tres grupos: $a$ ) relaciones y características generales de personalidad; $b$ ) características de integración de personalidad; por ejemplo, las tres dimensiones de personalidad de Eysenck: introversión- extroversión, psicoticismo y neuroticismo; $c$ ) el impulso o motivación; por ejemplo, la impulsividad y la búsqueda de sensaciones aventuradas.

Como puede observarse, en el comportamiento suicida interviene una serie de factores de naturaleza multicausal, ya sean aislados o bien derivados de los modelos previamente presentados, los cuales se relacionan con aspectos del intento de suicidio. Cabe señalar que dentro de estos factores los rasgos de personalidad parecen tener un rol importante cuando se busca entender el intento suicida. Dentro de las características de personalidad, un rasgo implicado en múltiples conductas de riesgo es la búsqueda de sensaciones (Cooper, Agocha y Sheldon, 2000; Cooper, Wood, Orcutt y Albino, 2003; Horvath y Zuckerman, 1993; Newcomb y McGee, 1991); a pesar de ello, se le ha dado poco interés en el momento de entender su relación con el intento de suicidio.

La búsqueda de sensaciones es un rasgo de personalidad que se define como la necesidad por experimentar variadas y complejas sensaciones, así como el deseo de correr riesgos físicos y sociales, por el simple deseo de disfrutar de tales experiencias; este concepto se considera multifacético y multidimensional, pues puede englobar componentes como la búsqueda de emoción y aventura, búsqueda de experiencias, desinhibición y susceptibilidad al aburrimiento (Zuckerman, 1994). El presente artículo utilizará esta definición en la evaluación de la búsqueda de sensaciones.

El término "búsqueda" se refiere a un rasgo que es expresado de forma activa y el de sensación al efecto sensorial de la estimulación externa que afecta al individuo. Este último es muy importante para definir su valor como reforzador primario, es decir, el estímulo externo que se busca para maximizar las sensaciones. Aquella persona que busca sensaciones, se caracteriza por realizar acciones inusuales, que para otros serían peligrosas y arriesgadas, (Zuckerman, 1979; 1994; Zuckerman y Kuhlman, 2000). Desde nuestra perspectiva, la búsqueda de sensaciones es un rasgo de 
personalidad con un fundamento bioconductual (Roberti, 2004; Zuckerman, 1994; 2007), debido a que, como se ha demostrado, la búsqueda de sensaciones es parte de patrones conductuales específicos. La evaluación de este rasgo permitirá identificar adolescentes que pretendan o deseen buscar novedad o estimulación.

\section{Búsqueda de sensaciones e intento de suicidio}

Existe evidencia que vincula la búsqueda de sensaciones con el intento de suicidio; por ejemplo, en los estudios sobre conducta suicida y búsqueda de novedad (Becerra, Paez, Robles-García y Vela, 2005; Ferguson, Beautrais y Horwood, 2003; Guillem, Pelissolo, Notides y Lépine, 2002) se encontró que ésta es mayor en personas que han intentado suicidarse en comparación con quienes no lo han intentado.

Bolognini, Laget, Plancherel, Atéphan, Corcos y Halfon (2002) estudiaron la relación entre el intento de suicidio y el abuso de drogas a partir de rasgos de personalidad. Los autores encontraron que las personas con intento de suicidio tuvieron un puntaje elevado en la escala de búsqueda de experiencias en comparación con los que no lo habían intentado; consideran que la búsqueda de sensaciones es una característica de las personas que presentan abuso de drogas. Asimismo (ambos, consumo de drogas y búsqueda de sensaciones) son predictores significativos en mujeres con intento de suicidio. Si bien se encuentra un efecto significativo de la búsqueda de sensaciones sobre el intento de suicidio, los autores no controlaron el efecto que puede tener al consumo de drogas sobre él, ya que hay jóvenes que intentan suicidarse sin haber consumido drogas o bien existen jóvenes que consumen drogas y no intentan suicidarse. De ahí la importancia de encontrar el efecto único que puede tener la búsqueda de sensaciones sobre el intento suicida.

Por otra parte, Vermeiren, Schwab-Stone, Ruchkin, King y Van Heeringen (2003) señalan que altos niveles de búsqueda de sensaciones se encuentran en hombres que han intentado suicidarse en comparación con 
los que no lo han intentado. Asimismo, se ha encontrado que las personas que no han intentado suicidarse difieren en su búsqueda de sensaciones de quienes lo han intentado una o más veces, específicamente en la dimensión de desinhibición. Para la dimensión de susceptibilidad al aburrimiento se encuentran diferencias entre quienes lo han intentado una vez y los que lo han intentado varias ocasiones; sin embargo, no existen diferencias significativas entre quienes lo han intentado y los que no (Laget, Plancherel, Stéphan, Bolognini, Corcos, Jeammet y Halfon, 2006).

\section{Justificación}

Por su prevalencia e incidencia en los jóvenes, el intento de suicidio se ha convertido en un problema de salud pública. Debido a ello, la investigación ha identificado los factores que afectan este comportamiento. La evidencia mostrada señala la interrelación entre la búsqueda de sensaciones y conductas como el consumo de alcohol, tabaco o drogas, entre otras, así como su influencia en la presencia de estos comportamientos. Sin embargo, existe poca evidencia que estudie de forma específica la búsqueda de sensaciones y el intento de suicidio. De manera adicional, los estudios que sí lo hacen no incorporan indicadores de letalidad, pues se ha documentado (Brown et al., 2004; González-Forteza et al., 2002; Nock et al., 2007) que la letalidad o el deseo de morir incide directamente en el intento de suicidio. Por tal razón, en el presente estudio se incorpora el deseo de morir como indicador de la letalidad del intento de suicidio. Se espera una asociación de los rasgos de personalidad (búsqueda de sensaciones) con la letalidad y el intento de suicidio.

Aunado a lo anterior, el estudio de la búsqueda de sensaciones con un nuevo instrumento de medición ofrece apoyo empírico a este constructo, debido a que la búsqueda de sensaciones como rasgo de personalidad permite definir qué tanto posee un individuo este rasgo y la forma característica de responder ante determinadas situaciones. Al mismo tiempo se contribuye con evidencia del efecto que tiene este rasgo en el intento 
de suicidio, puesto que en México no existen estudios que investiguen la relación entre la búsqueda de sensaciones y el intento suicida. Por lo anterior, los objetivos de esta investigación son describir las características del intento de suicidio, determinar las diferencias en la búsqueda de sensaciones entre los adolescentes que han y no han intentado suicidarse, así como conocer el grado de asociación entre la búsqueda de sensaciones y la letalidad (deseo de morir).

\section{Método}

\section{Participantes}

Se seleccionó una muestra no probabilística de 550 jóvenes, 260 hombres y 290 mujeres, con un rango de edad entre 14 y 23 años y una media de 16.88 años, estudiantes de dos escuelas públicas de educación media superior del Distrito Federal.

\section{INSTRUMENTO}

Para medir el intento de suicidio, se utilizaron las respuestas a los reactivos del instrumento de González-Forteza (2002), el cual se operacionalizó por medio de la respuesta afirmativa a la pregunta "¿jalguna vez a propósito te has hecho daño con el fin de quitarte la vida?", la edad del único/ último intento, así como, el motivo para hacerlo.

Para medir la búsqueda de sensaciones, se elaboró una escala que lleva el mismo nombre compuesta por 59 reactivos. Para obtener la validez de constructo y someter a prueba la estructura empírica subyacente, se realizó un análisis factorial exploratorio de componentes principales con rotación ortogonal para obtener la agrupación de los ítems. La solución factorial obtenida explica $55.8 \%$ de la varianza total, revelando ocho dimensiones: búsqueda de placer (14 reactivos, $\alpha=.925$, rango de 14 a 55 , por ejemplo, busco cosas placenteras), búsqueda de riesgo ( 9 reactivos, 
$\alpha=.906$, rango de 9 a 36 , por ejemplo, me gusta hacer cosas arriesgadas), búsqueda de emociones (8 reactivos, $\alpha=.866$, rango de 8 a 35 , por ejemplo, me gustan las emociones fuertes), búsqueda de novedad (7 reactivos, $\alpha=.864$, rango de 8 a 28 , por ejemplo, me gusta vivir experiencias nuevas) búsqueda de experiencias y aventura (8 reactivos, $\alpha=.834$, rango de 8 a 32, por ejemplo, me gustaría explorar lugares extraños), desinhibición (4 reactivos, $\alpha=.869$, rango de 4 a 16, por ejemplo, disfruto la compañía de personas desinhibidas), búsqueda de lo inusual ( 4 reactivos, $\alpha=.713$, rango de 4 a 16 , por ejemplo, me visto con un estilo extravagante), susceptibilidad al aburrimiento ( 5 reactivos, $\alpha=.766$, rango de 5 a 20, por ejemplo, me aburro si tengo que estar en mi casa). La escala cuenta con cuatro opciones de respuesta tipo Likert (nunca a siempre), la cual se encuentra fundamentada en el planteamiento teórico de Zuckerman (1979; 1991; 1994; 2007) y Roberti (2004).

\section{Procedimiento}

Se aplicó el instrumento a los adolescentes de manera grupal, utilizando los grupos escolares para tal fin. Se les pidió que respondieran un cuestionario elaborado para conocer algunas actividades que realizan jóvenes de su edad, sugiriéndoles que fueran honestos, porque la información proporcionada sería confidencial y se utilizaría sólo con fines de investigación. Su participación fue voluntaria y al final de la aplicación se entregó a cada participante una lista de números telefónicos de centros de apoyo psicológico.

\section{Resultados}

De los 550 adolescentes encuestados, el 12.5\% informó que ha intentado quitarse la vida. De éstos, el $28.9 \%$ fueron hombres y el $71 \%$ mujeres; a su vez el $15.9 \%$ de los hombres y el $46.3 \%$ de las mujeres que reportaron intento suicida, lo han hecho una sola vez. Asimismo el $13 \%$ de los varones y $24.6 \%$ de las mujeres lo han intentado dos o más ocasiones. La 
edad promedio del único o último intento fue de 15.28 (sd=1.6) años para el total de los adolescentes. En los varones, la edad de intento fue a los 15.65 (sd=1.7) años y en las mujeres fue a los 15.14 (sd= 1.5) años; es decir, la conducta suicida se llevó a cabo durante los años de secundaria. No se encontraron diferencias significativas en la edad del intento entre hombres y mujeres $(t=1.18, p=.241)$.

En cuanto a los motivos que llevaron a los jóvenes a cometer el intento suicida, se encontró que el motivo más frecuente corresponde a la dimensión emocional, de forma específica la soledad, la tristeza y la depresión, obteniendo el porcentaje mayor con $46.9 \%$, seguido de la incomprensión, falta de cariño y decepción (17.2\%) y, por último, una baja autoestima, tanto para hombres como para mujeres. En el caso de la esfera interpersonal, tanto para hombres como para mujeres, corresponde a los problemas familiares (17.2\%), y los problemas personales representan un 12.5\%. Respecto de los eventos que precipitaron el intento, se encontró, con $1.6 \%$, la ausencia de los padres y problemas escolares, y con 1.6\%, los problemas económicos.

Al tomar en cuenta $12.5 \%$ de adolescentes que intentaron quitarse la vida, el método utilizado con mayor frecuencia para efectuar el intento suicida fue utilizar algún objeto punzocortante (71.7\%). El segundo método utilizado fue la intoxicación con pastillas y/o medicamentos con un porcentaje de $20 \%$. El tercero consistió en aventarse $(5 \%$,). Por último, se encuentra el ahorcarse o asfixiarse, con 3.3\%, el cual se encontró sólo para los hombres. Con la finalidad de conocer si el intento suicida les había dejado alguna consecuencia, se indagó si fueron hospitalizados; sólo $1.5 \%$ respondió de manera afirmativa a esa pregunta. El indicador que evaluó el deseo de morir indicó que el $0.8 \%$ de hombres y $5.5 \%$ de mujeres no querían morir, $3.5 \%$ de hombres y $5.5 \%$ de mujeres no le importaba si moría o vivía y $3.5 \%$ de hombres y $5.9 \%$ de mujeres deseaba morir. Significativamente $(t=-2.53, p=.012)$, el deseo de morir es mayor en las mujeres $(\mathrm{M}=1.34$, $\mathrm{sd}=.83)$ que en los hombres $(\mathrm{M}=1.18$, $\mathrm{sd}=.65$, rango de 1-4) (ver tabla 1). Para analizar la relación entre el intento suicida y el grado de letalidad, se realizó una correlación de Pearson, encontrando una 
$r=.80, p<.001$, es decir, que existe un asociación positiva y significativa entre el intento de suicidio y el grado de letalidad.

Tabla 1. Letalidad del intento de suicidio por sexo

\begin{tabular}{|l|c|c|c|}
\hline Letalidad & Total & $\begin{array}{c}\text { Hombres* } \\
(n=20)\end{array}$ & $\begin{array}{c}\text { Mujeres* } \\
(n=49)\end{array}$ \\
\hline Nunca lo he hecho. & $87.5 \%$ & $92.3 \%$ & $83.1 \%$ \\
\hline No quería morir. & $3.3 \%$ & $0.8 \%$ & $5.5 \%$ \\
\hline $\begin{array}{l}\text { No les importaba } \\
\text { si vivían o morían. }\end{array}$ & $4.5 \%$ & $3.5 \%$ & $5.5 \%$ \\
\hline Deseaban morir. & $4.7 \%$ & $3.5 \%$ & $5.9 \%$ \\
\hline
\end{tabular}

*Porcentajes calculados sobre cada total de hombres y mujeres

Para realizar las comparaciones entre quienes habían intentado suicidarse y quienes no lo habían hecho, se recodificó la variable de intento de suicidio, de manera que las opciones una vez y más de una vez del intento suicida quedaron conformadas como el grupo que había realizado el intento suicida. Además se igualó la muestra por sexo y edad, con la finalidad de que quedaran grupos equivalentes ( $\mathrm{N}=69)$. Para analizar las diferencias en la búsqueda de sensaciones en los adolescentes que habían intentado suicidarse y los que no, se utilizó la prueba $t$ de Student.

La tabla 2 muestra que los jóvenes que han intentado suicidarse buscan más placer, riesgos y emociones intensas; les gusta realizar actividades novedosas, buscan experiencias y aventura, son desinhibidos y procuran hacer cosas inusuales, a diferencia de los jóvenes que no han intentado suicidarse. La susceptibilidad al aburrimiento no mostró ser diferente entre estos dos grupos. La característica de personalidad que se estimó en mayor proporción fue la búsqueda de riesgo $(\mathrm{OR}=1.1$, IC 95\%= 1.0- 1.1, $\mathrm{p}<.001$; pseudo $\mathrm{R}^{2}$ de Negelkerke=.15), es decir, los adolescentes que buscan riesgos tienen 1.1 veces mayor probabilidad de intentar suicidarse.

Con la finalidad de conocer el grado de asociación entre el indicador de letalidad (deseo de morir) y la búsqueda de sensaciones, se realizó una 
Tabla 2. Diferencias en el Intento de suicidio y la Búsqueda de Sensaciones

\begin{tabular}{|l|c|c|c|c|c|c|}
\hline & \multicolumn{2}{|c|}{$\begin{array}{c}\text { Con intento } \\
(N=69)\end{array}$} & \multicolumn{2}{c|}{$\begin{array}{c}\text { Sin intento } \\
(N=69)\end{array}$} & $t$ & $\eta^{2}$ \\
\hline & $\mathrm{M}$ & $\mathrm{SD}$ & $\mathrm{M}$ & $\mathrm{SD}$ & & \\
\hline Búsqueda de placer & 34.88 & 10.92 & 29.67 & 8.40 & $-3.15^{* *}$ & .06 \\
\hline Búsqueda de riesgo & 22.08 & 7.12 & 17.51 & 5.62 & $-4.19^{* *}$ & .11 \\
\hline $\begin{array}{l}\text { Búsqueda de } \\
\text { emociones intensas }\end{array}$ & 24.76 & 6.23 & 22.45 & 6.49 & $-2.13^{*}$ & .03 \\
\hline Búsqueda de novedad & 22.56 & 3.94 & 20.01 & 3.75 & $-3.90^{* *}$ & .10 \\
\hline $\begin{array}{l}\text { Búsqueda de experiencias } \\
\text { y aventura }\end{array}$ & 24.14 & 5.43 & 20.40 & 5.27 & $-4.12^{* *}$ & .11 \\
\hline Desinhibición & 10.82 & 3.72 & 9.37 & 2.97 & $-2.54^{* *}$ & .04 \\
\hline Búsqueda de lo inusual & 9.92 & 2.93 & 8.22 & 2.34 & $-3.77^{* *}$ & .09 \\
\hline $\begin{array}{l}\text { Susceptibilidad } \\
\text { al aburrimiento }\end{array}$ & 12.95 & 3.87 & 11.98 & 3.79 & -1.49 & .01 \\
\hline
\end{tabular}

$* p<.05 * * p<.01$

correlación de Pearson. Los resultados mostraron que el grado de letalidad se asoció positivamente con la búsqueda de placer $(r=.26, p<.001)$, de riesgo $(r=.30, p<.001)$, de emociones intensas $(r=.19, p<.05)$, de novedad $(r=.33, p<.001)$, de experiencias y aventura $(r=.31, p<.001)$, la desinhibición $(r=.17, p<.05)$ y de lo inusual $(r=.27, p<.001)$. La susceptibilidad al aburrimiento no mostró asociarse de forma significativa $(r=.10, p=.232)$ con el grado de letalidad.

\section{Discusión}

Los resultados obtenidos respecto de la frecuencia, edad y métodos del intento suicida coinciden con lo encontrado en otros estudios (Andrade et al., 2003; Cohen et al., 1996; González- Forteza et. al., 2002; INEGI, 2005; Palacios et al., 2006), pues se observa que las mujeres son las que intentan 
suicidarse con mayor frecuencia, al compararlas con los hombres. Asimismo, la edad promedio del único o último intento suicida fue alrededor de los 15 años. Los métodos más utilizado fueron mediante objetos punzocortantes e intoxicación con pastillas o medicamentos. Si se toma como base la edad promedio del intento suicida, así como las desviaciones estándar, puede considerarse que en este periodo de edad, los jóvenes cursaban los últimos años de la secundaria, por lo cual niveles educativos como la primaria y la secundaria constituyen sectores propicios para prevenir y atender la conducta suicida. Además, los últimos años de primaria resultan ser poblaciones idóneas para realizar programas de prevención, así como para promover una educación para la salud en dos sentidos: primero, con el fin de que se actúe antes de que aparezca la conducta suicida; segundo, dotar a los niños y adolescentes de recursos para el manejo de emociones como la tristeza, motivo principal de intento de suicidio, así como trabajar tales recursos desde el ámbito clínico, cuando la conducta ha ocurrido sin desenlace mortal y así evitar que vuelva a ocurrir.

En este estudio, el motivo más frecuente que llevó a los jóvenes a intentar suicidarse se encontró en la dimensión emocional: la soledad, la tristeza y la depresión, hallazgo diferente a los reportes tanto del INEGI (2005) — los cuales señalan que la primera causa de intento suicida se debe a disgustos familiares - como de otros autores (González-Forteza et al., 2002; Palacios, et al, 2006) quienes muestran como motivo principal los problemas familiares, correspondientes a la dimensión interpersonal. Tales diferencias pudieron deberse a la muestra seleccionada, a que en este grupo de adolescentes la dimensión emocional es un motivo más fuerte para intentar suicidarse que los motivos familiares o quizá porque se sienten más solos, tristes y deprimidos por los problemas familiares.

De acuerdo con los porcentajes obtenidos en cuanto al grado de letalidad, existen similitudes en comparación con el estudio de GonzálezForteza et al. (2002), quienes reportan que el porcentaje de aquellas personas que deseaban morir es ligeramente mayor en las mujeres, resultado similar a los hallazgos obtenidos en este estudio. La letalidad en el intento de suicidio cobra relevancia, pues el deseo de morir no necesariamente 
significa que va a producirse un daño con desenlace fatal; sin embargo, la suma de elementos como éste, junto con el objeto con el que se produce el daño (por ejemplo, una arma de fuego) incrementa la probabilidad y/o letalidad para consumar el acto.

Por otra parte, el análisis de medias señaló, a través del deseo de morir, que las mujeres muestran un mayor grado de letalidad en comparación con los hombres; a pesar de lo anterior, cuando se habla de letalidad por medio de los suicidios consumados, se destaca que son los varones quienes utilizan métodos más letales, según lo reportado por el INEGI (2005), respecto de los suicidios consumados (aunque no se señale el sexo de quien los cometió), así como lo señalado en estudios previos (Andrade et al., 2003; Cohen et al., 1996; Palacios et al., 2006); es decir, aun cuando la problemática del intento suicida predomina en la población femenina, siguen siendo los varones quienes más consiguen suicidarse. Como complemento de lo anterior, la evidencia mostrada deja claro que en los adolescentes con un mayor número de intento de suicidio, el deseo de morir es mayor, como se muestra en la correlación entre el deseo de morir y el intento suicida.

Con referencia a las comparaciones entre los adolescentes que han intentado suicidarse y los que no, los resultados presentados muestran evidencia de que los adolescentes con intento de suicidio presentan mayores niveles de búsqueda de sensaciones en comparación con los que no han cometido un acto suicida (Becerra et al., 2005; Bolognini et al., 2002; Ferguson et al., 2003; Guillem et al., 2002; Laget et al., 2006; Vermeiren et $a l ., 2003)$. Lo anterior permite entender que los jóvenes altos en búsqueda de sensaciones evalúan el riesgo como bajo. Esta disminución del riesgo aumenta la necesidad de experimentación e incrementa la probabilidad de que adolescentes en alta búsqueda de sensaciones tiendan a experimentar y a realizar actividades que dan la oportunidad de intentar quitarse la vida; es decir, es probable que los adolescentes con puntajes elevados en búsqueda de sensaciones no evaluaran al intento suicida como un gran daño, sino que sólo buscaban autolesionarse, incrementando el rango de nuevas y variadas conductas. 
El análisis por dimensión muestra que todas las de la búsqueda de sensaciones difieren entre quienes han intentado suicidarse y quienes no, excepto en la susceptibilidad al aburrimiento, diferente de lo reportado por Bolognini et al., (2002), quienes señalan que sólo la búsqueda de experiencia es un predictor importante del intento de suicidio en mujeres. La diferencia encontrada entre el estudio de Bolognini et al., (2002) y el nuestro puede deberse a que en el primero evaluaron a usuarios de drogas, variable que puede estar interviniendo al hacer los análisis, pues lo autores no controlaron el efecto que ésta pueda tener sobre el intento de suicidio. De ahí que las diferencias entre ambos estudios puede ser por la interacción entre el consumo de drogas, la búsqueda de sensaciones y el intento de suicidio.

Los resultados obtenidos ponen de manifiesto que buscar sensaciones es un importante correlato en la presencia del intento suicida. La comparación de medias (con tamaños de efecto de moderados a grandes, es decir, valores $\eta^{2}$ superiores a .03) permite mostrar evidencia que señala la importancia de estudiar este rasgo como un aspecto importante cuando se trabaja con adolescentes con intento de suicidios. Las distintas manifestaciones de este rasgo y el efecto sobre el intento de suicidio puede atribuirse a que la búsqueda de sensaciones es una característica de personas que les gusta correr riesgos (Bolognini et al., 2002; Guillem et al., 2002; Horvath et al., 1993; Rosenbloom, 2003), pues se propone que los individuos altos en búsqueda de sensaciones funcionan y se sienten mejor con un alto nivel de activación cortical; son motivados a realizar actividades que incrementan su activación sensorial (Newcomb et al., 1991; Zuckerman, 1991).

La evidencia antes mostrada permite explicar que en el intento de suicidio se incluye una alta búsqueda de novedad (Becerra et al., 2005; Bolognini et al., 2002; Guillem et al., 2002) y en estos individuos la búsqueda de sensaciones puede ser satisfecha por una variedad de actividades que estimulan los sentidos (Newcomb et al., 1991; Zuckerman, 1979; 1994; 2007). En este caso, hacerse daño a sí mismo (a), estimulando así sus sentidos y buscando nuevas experiencias, con la finalidad de sustituir el dolor emocional por un dolor físico a través de los sentidos. 
La presencia de la búsqueda de placer y la de emociones intensas en el intento de suicidio permite vislumbrar que el intento de suicidio es una forma de eliminar emociones negativas (probablemente depresión, ansiedad, enojo o estrés) (Beautrais, 1998; Goldston et al., 2006; GonzálezForteza et al., 2003; Spirito et al., 1995) y que hacerse daño a sí mismo permite ir en búsqueda de estímulos que provoquen placer (Bolognini et al., 2002; Guillem et al., 2002), debido a que la búsqueda de estímulos placenteros contribuye al riesgo de intentar suicidarse (Bolognini et al., 2002; Cohen et al., 1996; Guillem et al., 2002), es decir, el intento de suicidio podría ser el resultado de experimentar y probar algo nuevo o por una necesidad de sentir sensaciones novedosas (Cooper et al., 2003; Newcomb et al., 1991; Zuckerman, 1991; Zuckerman et al., 2000), lo cual los conduce a provocarse un daño a sí mismos.

Respecto de la dimensión de búsqueda de lo inusual, los adolescentes que presentan tentativa de suicidio muestran valores altos en este factor comparados con los que no lo han intentado; en otras palabras, los adolescentes con intento de suicidio, tienen un estilo extravagante, hacen cosas fuera de lo común —en este caso, intentar suicidarse— y buscan estímulos novedosos, como escuchar música nueva y poco común (Zuckerman, 1979; 1991). En este sentido, aquella persona que busca sensaciones, se caracteriza por realizar acciones inusuales y es más extrovertido (Zuckerman, 1979; 1994; Zuckerman et al., 2000). La manifestación de este rasgo permite entender que los adolescentes que intentan suicidarse expresan un estilo de vida no conformista. Realizar una tentativa de suicidio parece ser otra manifestación de inconformidad ya sea interna o externa. Al respecto, Cooper et al. (2000) señalan que las personas altas en extroversión se involucran en actividades de riesgo para sentir afecto y emociones positivas, debido a que son más susceptibles a las señales de recompensa. Otros autores (Loza et al., 1998; Roque et al., 2004) argumentan que los adolescentes que han intentado suicidarse son extravagantes y no miden el peligro.

En el presente estudio no se encontraron diferencias en la susceptibilidad al aburrimiento entre los adolescentes que han cometido alguna 
tentativa de suicidio y los que no. De manera similar a lo encontrado por Leget et al. (2006), quienes sólo encuentran diferencias en esta dimensión entre los que han intentado suicidarse una vez y los que lo han intentado varias veces, y no en los jóvenes que no lo han intentado comparados con los jóvenes que sí han intentado suicidarse.

En cuanto al grado de letalidad y la búsqueda de sensaciones, los hallazgos de este estudio muestran que aquellos adolescentes que han intentado suicidarse y que se describen como buscadores de placer, de riesgos, de emociones intensas; que manifiestan interés por experimentar cosas novedosas, buscar experiencias y aventura, ser más desinhibidos, así como hacer cosas inusuales, el deseo de morir es mayor. En la investigación previa sobre búsqueda de sensaciones e intento de suicidio no se había estudiado el grado de letalidad; la incorporación de este indicador en el presente estudio ayuda a conocer cómo la búsqueda de sensaciones se relaciona tanto con el intento como con el deseo de morir.

Lo anterior permite entender que cuando se estudia el intento de suicidio, la letalidad necesita ser evaluada como lo sugieren algunos autores (Brown et al., 2004; González-Forteza et al., 2002; Nock, et al., 2007), quienes señalan que la intención y la letalidad son elementos diferentes de las lesiones autoinflingidas y subdimensiones independientes del comportamiento suicida, pero no mutuamente excluyentes y, sin embargo, se complementan entre sí. Desde nuestra perspectiva, agregaríamos el método con el cual intentan suicidarse, como un elemento adicional en el estudio del intento suicida, de manera que estos tres elementos necesitan evaluarse para poder identificar con precisión a los posibles suicidas. La interacción entre la intención, el grado de letalidad y el método utilizado para atentar contra la vida pone de manifiesto que la conducta suicida en los adolescentes conlleva un riesgo en relación con las secuelas físicas y/o emocionales del intento. Además, revela que la problemática suicida está presente en la población escolar y, por lo mismo, necesita ser atendida (González-Forteza et al., 2002).

El intento de suicidio no sólo es una reacción a una variable específica (en este caso, la búsqueda de sensaciones como rasgo contribuye en cierto 
grado $\left[\eta^{2}=.11\right]$ a la presencia de este comportamiento), sino a varias, pues interviene, una serie de factores como se mostró en los modelos explicados anteriormente. Además, atentar contra la propia vida es una forma de evitar o escapar de una serie de dificultades en la vida de las personas. Algunos individuos no consiguen modificar lo que les afecta en su vida y por tanto recurren a esta opción de forma recurrente. En ambos casos, estos jóvenes se encuentran en un proceso de desajuste, para lo cual es necesario brindar toda la ayuda necesaria. Por esta razón, en el tratamiento de los individuos que han intentado suicidarse no sólo debe centrarse en los motivos o las intenciones, sino que deben indagarse las necesidades de estimulación (búsqueda de sensaciones) que llevaron a los adolescentes a intentar suicidarse. Lo anterior puede incluirse en una perspectiva cognitivo conductual, en donde el manejo de los recursos personales sea la base de la prevención y el tratamiento (Laget et al., 2006; Rivera, 2007).

\section{Conclusiones}

Como se ha descrito en la investigación sobre adolescentes, la búsqueda de sensaciones influye en una serie de conductas de riesgo y el intento de suicidio, como se ha mostrado en este estudio, no es la excepción. Los adolescentes con intento de suicidio registraron un puntaje superior en la búsqueda de sensaciones, manifestando conductas suicidas 1.1 veces más, a diferencia de quienes no lo han intentado quienes cuentan con menos niveles de búsqueda de sensaciones, valoran más el riesgo (como se observó, la búsqueda de riesgo es la dimensión que mejor estima este comportamiento) y/o cuentan con más recursos que les permite adaptarse a los problemas de la vida de forma distinta de los adolescentes altos en búsqueda de sensaciones y que han intentado suicidarse.

Identificar y reconocer a los adolescentes altos en búsqueda de sensaciones es un elemento importante a considerar en el campo de la prevención, debido a que la búsqueda de sensaciones ha sido identificada como un importante factor de riesgo en el consumo de sustancias adictivas y de 
la conducta antisocial. Si logra entenderse cómo en la búsqueda de riesgo se encuentra implicada la necesidad de estimulación sensorial externa podremos entender que quienes buscan sensaciones se caracterizan por llevar a cabo acciones, que para otros son peligrosas y arriesgadas, características presentes en el intento de suicidio. Con base en lo encontrado, como medida preventiva debe promoverse y fortalecerse en los jóvenes recursos o destrezas que les ayuden a controlar situaciones adversas de su vida cotidiana, generando en ellos la capacidad de controlar y manejar situaciones problema, valorar los riesgos y no responder ante la adversidad con respuestas inadecuadas (hacerse un daño a sí mismo).

Por último, debe tenerse en cuenta que esos resultados no pueden generalizarse a toda la población adolescente, sino que reflejan lo que está pasando con una muestra de jóvenes de educación media superior. Para corroborar tanto la estructura de la nueva escala de búsqueda de sensaciones, como las diferencias en el intento de suicidio, podrían hacerse comparaciones con muestras clínicas y confirmar los hallazgos obtenidos en este estudio.

\section{Agradecimientos}

Los autores agradecen a la Dra. Lilia Bertha Alfaro Martínez su apoyo en la aplicación de los cuestionarios y su interés en la presente investigación, así como a las autoridades de la Escuela Nacional Preparatoria que brindaron todas las facilidades para realizar este estudio.

Bibliografía

Andrade, P. P., O. D. Betancourt y V. M. Camacho (2003). Ambiente familiar de adolescentes que han intentado suicidarse. En Revista de Psicología Social y Personalidad, 19, pp. 55- 64.

Beautrais, A. (1998). Risk factors for serious suicide attempts among young people. En R. J. Kosky, H. S. Eshkevari, R. D. Goldney \& R. Hassan (eds.), Suicide Prevention, the Global Context. Nueva York: Plenum Press, pp. 167- 180. 
Becerra, B., F. Paez, R. Robles-García y G. E. Vela (2005). Perfil de temperamento y carácter de personas con intento suicida. En Actas Españolas de Psiquiatría, 33 (2), pp. 117-122.

Bolognini, M., J. Laget, B. Plancherel, P. Stéphan, M. Corcos \& O. Halfon (2002). Drug use and suicide attempts: the role of personality factors. En Substance Use \& Misuse, 37 (3), pp. 337-356.

Brown, G. K., G. R. Henriques, D. Sosdjan \& A. Beck (2004). Suicide intent and accurate expectations of lethality: Predictors of medical lethality of suicide attempts. En Journal of Consulting and Clinical Psychology, 72 (6), pp. 1170-1174.

Cohen, Y., A. Spirito y L. Brown (1996). Suicide and suicidal behavior. En R. J. DiClemente, W. B. Hansen y P. E. Lynn (eds.). Handbook of Adolescent Health Risk Behavior. Nueva York y Londres: Plenum Press, pp. 193- 217.

Cooper, M. L., V. D. Agocha \& M. S. Sheldon (2000). A motivational perspective on risky behaviors: The role of personality and affect regulatory processes. En Journal of Personality, 68, pp. 1059-1088.

, P. K. Wood, H. K. Orcutt \& A. Albino (2003). Personality and the predisposition to engage in risky or problem behavior during adolescence. En Journal of Personality and Social Psychology, 84, pp. 390-410.

Ferguson, D. M., A. L. Beautrais \& L. J. Horwood (2003). Vulnerability and resiliency to suicidal behaviours in young people. En Psychological Medicine, 33, pp. 61-73.

Goldston, D. B., B. A. Reboussin \& S. S. Daniel (2006). Predictors of suicide attempts: state and trait components. En Journal of Abnormal Psychology, 115 (4), pp. 842-849.

González-Forteza, C., G. S. Berenson, G. A. Tello, F. D. Facio y M. Medina-Mora (1998). Ideación suicida y características asociadas en mujeres adolescentes. En Salud Pública de México, 40, pp. 430-437.

, M. Mariño, E. Rojas, L. Mondragón y M. Medina (1998). Intento de suicidio en estudiantes de la ciudad de Pachuca, Hgo. y su relación con el malestar depresivo y el uso de sustancias. En Revista Mexicana de Psicología, 15 (2), pp. 165-175.

, C., L. L. Ramos, G. A. Caballero y E. F. Wagner (2003). Correlatos psicosociales de depresión, ideación e intento suicida en adolescentes mexicanos. En Psicothema, 15 (4), pp. 524-532.

, V. J. Villatoro, E. I. Alcántar, Ma. E. Medina-Mora, B. C. Fleiz, L. P. Bermúdez y B. N. Amador (2002). Prevalencia de intento suicida en estu- 
diantes adolescentes de la ciudad de México: 1997 y 2000. En Salud Mental, 26 (6), pp. 1-12.

Guillem, E., A. Pelissolo, C. Notides \& J. Lepine (2002). Relationship between attempted suicide, serum cholesterol level and novelty seeking in psychiatric in-patients. En Psychiatry Research, 112, pp. 83-88.

Harrington, R. (2001). Consecuencias psicosociales de la depresión adolescente. En Psiquiatría y Salud Integral, 1 (2), pp. 48-52.

Horesh, N., D. Gothelf, H. Ofek, T. Weizman \& A. Apter (1999). Impulsivity as a correlate of suicidal behavior in adolescent psychiatric inpatients. En Crisis, 20 (1), pp. 8-14.

Horvath, P. \& M. Zuckerman (1993). Sensation seeking, risk appraisal, and risky behavior. En Personality and Individual Differences, 14, pp. 41-52.

Instituto Nacional de Estadística, Geografía e Informática (2005). Estadísticas de Intentos de Suicidio y Suicidios. México: Serie Boletín de Estadísticas Continuas, Demográficas y Sociales, pp. 1-17.

Kaslow, N. J., M. P. Thompson, A. Okun, A. Price, S. Young, M. Bender, S. T. Wyckoff, H. Twomey, J. Goldin \& R. Parker (2002). Risk and protective factors for suicidal behavior in abused African American women. En Journal of Consulting and Clinical Psychology, 70, pp. 311-319.

Laget, J., P. B. Plancherel, P. Stéphan, M. Bolognini, M. Corcos, P. Jeammet \& O. Halfon (2006). Personality and repeated suicide attempts in dependent adolescents and young adults. En Crisis, 27(4), pp. 164-171.

Loza C. G., G. E. Lucio, P. C. y Durán (1998). Comparación entre la personalidad de un adolescente con intento suicida y sin intento suicida. En La Psicología Social en México, 8, pp. 80-85.

Newcomb, M. \& L. McGee (1991). Influence of sensation seeking on general deviance and specific problem behaviors from adolescence to young adulthood. En Journal of Personality and Social Psychology, 61, pp. 614-628.

Nock, M. K., E. B. Holmberg, V. I. Photos \& B. D. Michel (2007). Self-injurious thoughts and behaviors interview: Development, reliability and validity in an adolescent sample. En Psychological Assessment, 19 (3), pp. 309- 317.

Palacios, D. J., P. P. Andrade y O. D. Betancourt (2006). Intento de suicidio y consumo de alcohol en adolescentes. En A. R. Sánchez, R. Díaz-Loving y A. S. Rivera (eds.). En La Psicología Social en México, vol. 11, México: AmePso, pp. 174-180.

Rivera, H. M. E. (2007). Mensajes audiovisuales para la promoción de la salud y la prevención del suicidio en los adolescentes. Tesis de Doctorado, México, Universidad Nacional Autónoma de México. 
y P. P. Andrade (2006). Recursos individuales y familiares que protegen al adolescente del intento suicida. En Revista Intercontinental de Psicología y Educación, 8(2), pp. 23-40.

Roberti, J. W. (2004). A review of behavioral and biological correlates of sensation seeking. En Journal of Research in Personality, pp. 256-279.

Roque, Q. M., F. I. Valadez, J. M. González de Mendoza, M. J. Vega, R. L. Flores y A. S. Valencia (2004). Tipos de personalidad y conducta suicida. En Investigación en Salud, 6 (2), pp. 108-113.

Rosenbloom, T. (2003). Risk evaluation and risky behaviors of high and low sensation seekers. En Social Behavior and Personality, 31, pp. 375-386.

Spann, M., S. D. Molock, C. Barksdale, S. Matlin \& R. Puri (2006). Suicide and African American teenagers: risk factors and coping mechanisms. En Suicide and Life-Threatening Behavior, 36 (5), pp. 553-568.

Spirito, A., J. Overholser \& L. Vinnick (1995). Adolescent suicide attempters in general hospitals: psychological evaluation and disposition planning. En J. Wallander \& J. S. Lawrence (eds.). Adolescent Health Problems: Behavioral Perspectives. Nueva York: Gilford Press, pp. 97-116.

Vermeiren, R., M. Schwab-Stone, V. Ruchkin, R. King \& C. Van Heeringen, D. Deboutte (2003). Suicidal behavior and violence in male adolescents: A school-based study. En Journal of the American Academy of Child \& Adolescent Psychiatry, 42, pp. 41-48.

Stoelb, M. \& J. Chiriboga (1998). A process model for assessing adolescent risk for suicide. En Journal of Adolescence, 21, pp. 359-370.

Zuckerman, M. (1979). Sensation seeking: beyond the optimal level of arousal. Hillsdale, Nueva Jersey: LEA.

(1991). Psychobiology of personality. Massachusetts: Cambridge University Press.

(1994). Behavioral expressions and biosocial bases of sensation seeking. Massachusetts: Cambridge University Press.

- (2007). Sensation seeking and risky behavior. Washington: American Psychological Association.

y D. M. Kuhlman (2000). Personality and risk taking: Common biosocial factors. En Journal of Personality, 68, pp. 999-1029. 